\title{
Power Series Solution of the Hamilton-Jacobi-Bellman Equation for Descriptor Systems
}

\author{
Johan Sjöberg, Torkel Glad \\ Division of Automatic Control \\ Department of Electrical Engineering \\ Linköpings universitet, SE-581 83 Linköping, Sweden \\ WWW: http://WwW. control.isy.liu.se \\ E-mail: johans@isy.liu.se, torkel@isy.liu.se
}

21st September 2005

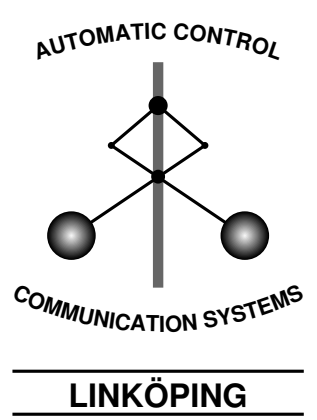

Report no.: LiTH-ISY-R-2701

Submitted to CDC 2005, Sevilla, Spain

Technical reports from the Control \& Communication group in Linköping are available at http://www.control.isy.liu.se/publications. 


\begin{abstract}
Optimal control problems for a class of nonlinear descriptor systems are considered. It is shown that they possess a well-defined analytical feedback solution in a neighborhood of the origin, provided stabilizability and some other regularity conditions are satisfied. Explicit formulas for the series expansions of the cost function and control law are given.
\end{abstract}

Keywords: Optimal Control, Descriptor Systems 


\section{Power Series Solution of the Hamilton-Jacobi-Bellman Equation for Descriptor Systems}

\author{
Johan Sjöberg \\ Division of Automatic Control \\ Department of Electrical Engineering \\ Linköpings universitet, SE-581 83 Linköping, SWEDEN \\ johanseisy.liu.se
}

\author{
Torkel Glad \\ Division of Automatic Control \\ Department of Electrical Engineering \\ Linköpings universitet, SE-581 83 Linköping, SWEDEN \\ torkel@isy.liu.se
}

\begin{abstract}
Optimal control problems for a class of nonlinear descriptor systems are considered. It is shown that they possess a well-defined analytical feedback solution in a neighborhood of the origin, provided stabilizability and some other regularity conditions are satisfied. Explicit formulas for the series expansions of the cost function and control law are given.
\end{abstract}

\section{INTRODUCTION}

This paper deals with the control problem of nonlinear descriptor systems. This area has also been studied in for example [1], [2], [3]. More specificly, we focus on optimal feedback control and the goal is to find an approximate solution. For state space systems this problem was first considered in [4]. There it was shown that the solution to the optimal control problem could be obtained in form of power series, the terms of which could be sequentially obtained through solution of a quadratic optimal control problem for the linearized system and subsequent solution of a series of linear partial differential equations. Further, a formal proof of the convergence of the power series was presented in the case when the input signal is scalar and the system has the form $\dot{x}=f(x)+B u$. In [5] these results were extended to general state space systems, $\dot{x}=f(x, u)$, and this work was extended even more in [6]. In the earlier works [4], [5], the functions involved were required to be analytic functions around the origin. In [6], this requirement was relaxed to twice differentiability. For a good overview of more recent methods to find approximative solutions of the HamiltonJacobi-Bellman equation, see [7] and references therein.

The optimal control problem considered in this paper is defined in terms of a descriptor system in $\mathbb{R}^{n}$

$$
E \dot{x}=F(x, u)
$$

and a performance integral

$$
V(E x)=\int_{0}^{\infty} L(x, u) d t
$$

The objective is to find a function, $u(x) \in \mathbb{R}^{d}$, which in a neighborhood of the origin, $(x, u)=(0,0)$, stabilizes (1), i.e., makes

$$
E \dot{x}=F(x, u(x))
$$

locally asymptotically stable and minimizes (2) given some initial conditions, $E x(0)=E x_{0}$, close enough to the origin. In the descriptor system case it is also necessary to require that the system (1) is made impulse free by $u(x)$.
Notation: The notation in this paper is fairly standard. The Jacobian matrix $\frac{\partial h}{\partial x}$ will be denoted $h_{x}$ and $(\cdot)^{(i)}$ will be used to denote the terms of order $i$ in a power series expansion. $Q \succ 0$ means that $Q$ is a real positive definite matrix. $\lfloor m\rfloor$ will denote the integer part of $m$.

\section{BASIC ASSUMPTIONS}

Throughout this paper some assumptions are made. The nonlinear descriptor system is assumed to be partitioned as

$$
\begin{aligned}
\dot{x}_{1} & =f_{1}\left(x_{1}, x_{2}, u\right) \\
0 & =f_{2}\left(x_{1}, x_{2}, u\right)
\end{aligned}
$$

where $x_{1} \in \mathbb{R}^{r}, x_{2} \in \mathbb{R}^{n-r}$ and $u \in \mathbb{R}^{d}$. Further, the origin is assumed to be a stationary point, i.e., $F(0,0)=0$.

The functions $F(x, u)$ and $L(x, u)$ are assumed to be analytic functions in some neighborhood of the origin, $x=$ $0, u=0$, which implies that they can be expanded in convergent power series

$$
\begin{aligned}
F(x, u) & =A x+B u+F_{h}(x, u) \\
L(x, u) & =x^{T} Q x+2 x^{T} S u+u^{T} R u+L_{h}(x, u)
\end{aligned}
$$

where the matrices $A, B, Q, S$ are partitioned as

$$
\begin{aligned}
A=\left(\begin{array}{ll}
A_{11} & A_{12} \\
A_{21} & A_{22}
\end{array}\right), & B=\left(\begin{array}{l}
B_{1} \\
B_{2}
\end{array}\right) \\
Q=\left(\begin{array}{ll}
Q_{11} & Q_{12} \\
Q_{21} & Q_{22}
\end{array}\right), & S=\left(\begin{array}{l}
S_{1} \\
S_{2}
\end{array}\right)
\end{aligned}
$$

and $F_{h}(x, u)$ and $L_{h}(x, u)$ contain higher order terms of at least degree two and three respectively.

The method described in this paper will require that $A_{22}$ in (5a) is nonsingular which is equivalent to that

$$
\operatorname{rank} \frac{\partial f_{2}}{\partial x_{2}}(0,0,0)=n-r
$$

This is implied if the system (1) is of index one [8].

Remark 1: The index one assumption does not need to restrict the usability of the results in this work too much. In the paper [9] it has been shown how to locally rewrite a general, possibly high index, nonlinear descriptor system to an index one system. Furthermore, in many cases, for example for mechanical multibody systems, the resulting system is on semi-explicit form.

The initial conditions will be assumed to be consistent, i.e., that $f_{2}\left(x_{1}(0), x_{2}(0), u(0)\right)=0$. 
For the performance criterion (2) to converge, it is necessary that the state converges towards the origin fast enough and that the system shows no impulsive behavior in the solution. In this paper we will rewrite the descriptor system as a state space system. Therefore, no impulsive behavior will occur. Under the assumption that the initial conditions are chosen close enough to the origin, local asymptotic stability of the linearized closed loop system (3) will yield that the solution converges exponentially, which is sufficiently fast. The considered feedback law can be expressed as

$$
u(x)=D x+u_{h}(x)
$$

where $u_{h}(x)$ consists of terms of degree two or higher. After rewriting the linearization of the descriptor system as a linear state space system we obtain

$$
\dot{x}=(\hat{A}+\hat{B} D) x
$$

where $\hat{A}, \hat{B}$ are some matrices and $D$ is given in (7). This system has to be asymptotically stable and therefore, a fundamental assumption is the existence of a feedback law such that $\Re \lambda(\hat{A}+\hat{B} D)<0$. This is equivalent to $(\hat{A}, \hat{B})$ being stabilizable. A system matrix for which all eigenvalues have strictly negative real parts is denoted Hurwitz.

\section{Review of Series Solutions to the Optimal CONTROL PROBleM}

For systems on state space form, i.e., when the $E$ matrix is an identity matrix, it is well-known (see for example [10]) that the optimal control problem mentioned in Section I is associated with the Hamilton-Jacobi-Bellman equation (HJB)

$$
0=\min _{u(\cdot)} L(x, u)+V_{x}(x) F(x, u)
$$

The HJB is a partial differential equation, which often is difficult to solve explicitly even for small systems. Therefore, [4], [5], [6] derived series solutions of the HJB near the origin. The idea is that if $L(x, u), F(x, u)$ and $u(x)$ are analytical near the origin and if $u(x)$ is chosen such that it stabilizes the closed loop system locally around $(x, u)=0$ the cost function $V(x)$ will be analytical in a neighborhood of the origin [6]. This guarantees that $V(x)$ can be expressed as a convergent power series

$$
V(x)=x^{T} P x+V_{h}(x)
$$

where $V_{h}(x)$ contains the terms of order three and higher. The HJB is locally around the origin equivalent to two equations [6], namely

$$
\begin{aligned}
& 0=L\left(x, u_{*}(x)\right)+V_{x}(x) F\left(x, u_{*}(x)\right) \\
& 0=L_{u}\left(x, u_{*}(x)\right)+V_{x}(x) F_{u}\left(x, u_{*}(x)\right)
\end{aligned}
$$

where $u_{*}(x)$ is the optimal feedback law. If (5), (7) and (9) are inserted into (10), the result is two power series in $x$. Equation (10) holds for all $x$ near the origin, which implies that different orders of $x$ yield separate equations in the corresponding coefficients. The linear terms of the power series yield the equations [6]

$$
\begin{aligned}
P \tilde{A}+\tilde{A}^{T} P-P B R^{-1} B^{T} P+Q-S R^{-1} S^{T} & =0 \\
D_{*}+R^{-1}\left(S^{T}+B^{T} P\right) & =0
\end{aligned}
$$

where $\tilde{A}=A-B R^{-1} S^{T}$ while the higher order terms in $x$ will be

$$
\begin{aligned}
& V_{x}^{(m)}(x) A_{c} x=-\sum_{k=3}^{m-1} V_{x}^{(k)}(x) B u_{*}^{(m-k+1)}(x) \\
& -\sum_{k=2}^{m-1} V_{x}^{(k)}(x) F_{h}^{(m-k+1)}\left(x, u_{*}\right)-L_{h}^{(m)}\left(x, u_{*}\right) \\
& -2 \sum_{k=2}^{\left\lfloor\frac{m-1}{2}\right\rfloor} u_{*}^{(k)}(x)^{T} R u_{*}^{(m-k)}(x)-u_{*}^{(m / 2)}(x)^{T} R u_{*}^{(m / 2)}(x)
\end{aligned}
$$

where $m=3,4, \ldots$ and $A_{c}=A-B D_{*}$, and

$$
\begin{aligned}
& u_{*}^{(k)}(x)=-\frac{1}{2} R^{-1}\left\{V_{x}^{(k+1)}(x) B\right. \\
& \left.+\sum_{i=1}^{k-1} V_{x}^{(k-i+1)}(x) F_{h, u}^{(i)}\left(x, u_{*}\right)+L_{h, u}^{(k)}\left(x, u_{*}\right)\right\}
\end{aligned}
$$

for $k=2,3, \ldots$ In (12) the convention that $\sum_{k}^{l}=0$ for $l<k$ is used and the terms $u^{(m / 2)}$ are to be omitted if $m$ is odd. We see that the first terms of $u_{*}(x)$ and $V(x)$,

$$
u_{*}^{(1)}(x)=D_{*} x, \quad V^{(2)}(x)=x^{T} P x
$$

are given by the solution to an algebraic Riccati equation (ARE). Hence, the existence of a first order approximative optimal solution is guaranteed if there exists a unique positive definite stabilizing solution $P$ to the ARE. A theorem that tells us when this is fulfilled is given below.

Theorem 1: Consider the ARE (11a). Assume that the weight matrix satisfies $\left(\begin{array}{cc}Q & S \\ S^{T} & R\end{array}\right) \succ 0$. Then the following statements are equivalent:

1) $(A, B)$ is stabilizable.

2) The ARE (11a) has a unique positive definite stabilizing solution, i.e., a solution such that $A+B D_{*}$ is Hurwitz, where $D_{*}$ is given by (11b).

Proof: See [11].

In order to obtain higher order approximations of the optimal feedback law, $u_{*}(x)$, and the corresponding cost function, $V(x)$, we need to solve (12). First note that

$$
\begin{aligned}
F_{h}^{(k)}\left(x, u_{*}\right) & =F_{h}^{(k)}\left(x, u_{*}^{(1)}+u_{*}^{(2)}+\ldots+u_{*}^{(k-1)}\right) \\
L_{h}^{(k)}\left(x, u_{*}\right) & =L_{h}^{(k)}\left(x, u_{*}^{(1)}+u_{*}^{(2)}+\ldots+u_{*}^{(k-2)}\right)
\end{aligned}
$$

since $F_{h}(x, u)$ and $L_{h}(x, u)$ are power series beginning with terms of order two and three respectively. Based on this it can be seen that the right-hand side of (12a) only depends on the terms

$$
u_{*}^{(1)}, \ldots, u_{*}^{(m-2)}, V^{(2)}, \ldots, V^{(m-1)}
$$

and the right-hand side of (12b) only depends on

$$
u_{*}^{(1)}, \ldots, u_{*}^{(k-1)}, V^{(2)}, \ldots, V^{(k+1)}
$$

Since $A_{c}$ is Hurwitz, it is shown in for example [12], that the partial differential equation (12a) is uniquely solvable. Therefore, by starting with $u_{*}^{(1)}(x)=D_{*} x$ and $V^{(2)}(x)=$ $x^{T} P x$ it is possible to consecutively calculate the terms

$$
V^{(3)}(x), u_{*}^{(2)}(x), V^{(4)}(x), u_{*}^{(3)}(x), \ldots
$$

and thereby generating power series for $u_{*}(x)$ and $V(x)$. 


\section{The Descriptor System CAse}

In the descriptor case the matrix $E$ in (1) is singular. This means that the optimal control problem for descriptor systems is not associated with the ordinary HJB (8). However, in the case when (6) holds, i.e., when the linearized descriptor system has index one, it is possible to solve the constraint equation (4b) for $x_{2}$ using the implicit function theorem.

Theorem 2: Let $F(x, y): \mathbb{C}^{m} \times \mathbb{C}^{n} \mapsto \mathbb{C}^{m}$ be an analytic function of $(x, y)=\left(x_{1}, x_{2}, \ldots, x_{m}, y_{1}, y_{2}, \ldots, y_{n}\right)$ in a neighborhood of a point, $\left(x^{0}, y^{0}\right)$, and assume that $F\left(x^{0}, y^{0}\right)=0$ and that the matrix $F_{x}\left(x^{0}, y^{0}\right)$ is nonsingular. Then the equation $F(x, y)=0$ has a uniquely determined analytic solution

$$
x=\varphi(y)
$$

in a neighborhood of $y^{0}$, such that $\varphi\left(y^{0}\right)=x^{0}$.

Proof: See [13].

If the implicit function theorem is applied to $f_{2}\left(x_{1}, x_{2}, u\right)$ in a neighborhood of $\left(x_{1}^{0}, x_{2}^{0}, u^{0}\right)=(0,0,0)$ and with the variables chosen as $x=x_{2}$ and $y=\left(x_{1}, u\right), x_{2}$ can locally be expressed as

$$
x_{2}=\varphi\left(x_{1}, u\right)
$$

where $\varphi\left(x_{1}, u\right)$ is an analytical function with $\varphi(0,0)=0$. Using (16) it is possible to reduce the optimal control problem for the descriptor system to an optimal control problem for a state space system with some constraints on the initial conditions

$$
f_{2}\left(x_{1}(0), x_{2}(0), u(0)\right)=0
$$

The state space system will be given by

$$
\dot{x}_{1}=\hat{f}_{1}\left(x_{1}, u\right)=f_{1}\left(x_{1}, \varphi\left(x_{1}, u\right), u\right)
$$

and the cost function is reduced to

$$
\hat{L}\left(x_{1}, u\right)=L\left(x_{1}, \varphi\left(x_{1}, u\right), u\right)
$$

The optimal control problem is thus transformed to an ordinary problem in the state variables $x_{1}$, and the problem is solved by the HJB (8). Finding approximative solutions in this case is done by the method described in Section III.

However, a problem is that in many cases it is hard or even impossible to find an explicit expression for $\varphi\left(x_{1}, u\right)$. However, in order to calculate the approximative solutions of $V(x)$ and $u_{*}(x)$, using the method described in Section III, only the series expansions of the functions involved, i.e., $F(x, u), L(x, u)$ and $u(x)$, are needed. Thus, in order to determine an approximative solution to the descriptor problem the series expansions around $\left(x_{1}, u\right)=(0,0)$ of $\hat{f}\left(x_{1}, u\right)$ and $\hat{L}\left(x_{1}, u\right)$ in (17) are needed. The idea, used in this paper, is to utilize that the series expansions of the composite functions $\hat{f}_{1}\left(x_{1}, u\right)$ and $\hat{L}\left(x_{1}, u\right)$ can be computed based on the series expansions of $f_{1}\left(x_{1}, x_{2}, u\right), L\left(x_{1}, x_{2}, u\right)$, $u\left(x_{1}\right)$ and $\varphi\left(x_{1}, u\right)$. Therefore, the power series of $\varphi\left(x_{1}, u\right)$ is needed. From Theorem 2 it is known that $\varphi\left(x_{1}, u\right)$ is an analytical function in a neighborhood of origin, and therefore it has a convergent power series expansion. This series expansion is possible to compute recursively using (4b). Assume that

$$
x_{2}=\varphi\left(x_{1}, u\right)=\varphi^{(1)}\left(x_{1}, u\right)+\varphi_{h}\left(x_{1}, u\right)
$$

where $\varphi_{h}\left(x_{1}, u\right)$ contains terms beginning with degree two. From (5) we have that the series expansion of (4b) is given by

$$
\begin{aligned}
0 & =f_{2}\left(x_{1}, x_{2}, u\right) \\
& =A_{21} x_{1}+A_{22} x_{2}+B_{2} u+f_{2 h}\left(x_{1}, x_{2}, u\right)
\end{aligned}
$$

If (18) is combined with (19) the expression obtained is

$$
\begin{aligned}
0 & =A_{21} x_{1}+A_{22}\left\{\varphi^{(1)}\left(x_{1}, u\right)+\varphi_{h}\left(x_{1}, u\right)\right\}+B_{2} u \\
& +f_{2 h}\left(x_{1}, \varphi^{(1)}\left(x_{1}, u\right)+\varphi_{h}\left(x_{1}, u\right), u\right)
\end{aligned}
$$

Since (20) must hold for all $\left(x_{1}, u\right)$ close to the origin the first order term of $\varphi\left(x_{1}, u\right)$ will be given by

$$
\varphi^{(1)}\left(x_{1}, u\right)=-A_{22}^{-1} A_{21} x_{1}-A_{22}^{-1} B_{2} u
$$

since all other terms have degrees higher than one. Furthermore, since the lowest degree of the terms in $f_{2 h}\left(x_{1}, x_{2}, u\right)$ is two, we know that

$$
\begin{aligned}
& f_{2 h}^{(m)}\left(x_{1}, \varphi\left(x_{1}, u\right), u\right)= \\
& \quad f_{2 h}^{(m)}\left(x_{1}, \varphi^{(1)}\left(x_{1}, u\right)+\ldots+\varphi^{(m-1)}\left(x_{1}, u\right), u\right)
\end{aligned}
$$

This makes it is possible to derive a recursive expression for a general degree term of $\varphi\left(x_{1}, u\right)$ as

$$
\varphi^{(m)}\left(x_{1}, u\right)=-A_{22}^{-1} f_{2 h}^{(m)}\left(x_{1}, \varphi\left(x_{1}, u\right), u\right)
$$

Since the first term in $x_{2}$ is given by (21), the first order approximation will define a change of variables locally around the origin given by

$$
\left(\begin{array}{c}
x_{1} \\
x_{2} \\
u
\end{array}\right)=\Pi\left(\begin{array}{c}
x_{1} \\
u
\end{array}\right)=\left(\begin{array}{cc}
I & 0 \\
-A_{22}^{-1} A_{21} & -A_{22}^{-1} B_{2} \\
0 & I
\end{array}\right)\left(\begin{array}{c}
x_{1} \\
u
\end{array}\right)
$$

If (24) is applied to (5a) the system (17a) becomes

$$
\dot{x}_{1}=\hat{A} x_{1}+\hat{B} u+\hat{f}_{1 h}\left(x_{1}, u\right)
$$

where $\hat{A}=A_{11}-A_{12} A_{22}^{-1} A_{21}, \hat{B}=B_{1}-A_{12} A_{22}^{-1} B_{2}$ and

$$
\hat{f}_{1 h}\left(x_{1}, u\right)=f_{1 h}\left(x_{1}, \varphi\left(x_{1}, u\right), u\right)+A_{12} \varphi_{h}\left(x_{1}, u\right)
$$

We assume that the weight matrix is given in the original coordinates, i.e., $x_{1}, x_{2}$ and $u$, and not in the new coordinates $x_{1}$ and $u$. Therefore, the series expansion for (17b) becomes

$$
\begin{aligned}
\hat{L}\left(x_{1}, u\right) & =\left(\begin{array}{c}
x_{1} \\
u
\end{array}\right)^{T} \Pi^{T}\left(\begin{array}{cc}
Q & S \\
S^{T} & R
\end{array}\right) \Pi\left(\begin{array}{c}
x_{1} \\
u
\end{array}\right)+\hat{L}_{h}\left(x_{1}, u\right) \\
& =\left(\begin{array}{c}
x_{1} \\
u
\end{array}\right)^{T}\left(\begin{array}{cc}
\hat{Q} & \hat{S} \\
\hat{S}^{T} & \hat{R}
\end{array}\right)\left(\begin{array}{c}
x_{1} \\
u
\end{array}\right)+\hat{L}_{h}\left(x_{1}, u\right)
\end{aligned}
$$

where

$$
\begin{gathered}
\hat{L}_{h}\left(x_{1}, u\right)=L_{h}\left(x_{1}, \varphi\left(x_{1}, u\right), u\right)+2 x_{1}^{T} Q_{12} \varphi_{h}\left(x_{1}, u\right) \\
+2 \varphi^{(1)}\left(x_{1}, u\right) Q_{22} \varphi_{h}\left(x_{1}, u\right) \\
+\varphi_{h}\left(x_{1}, u\right)^{T} Q_{22} \varphi_{h}\left(x_{1}, u\right)+2 u^{T} S_{2} \varphi_{h}\left(x_{1}, u\right)
\end{gathered}
$$

Using the first order terms of the series expansions (25) and (27), the ARE (11a) and the expression for the first order 
term in the feedback (11b) for the descriptor system will become

$$
\begin{aligned}
P \tilde{\hat{A}}+\tilde{\hat{A}}^{T} P-P \hat{B} \hat{R}^{-1} \hat{B}^{T} P+\hat{Q}-\hat{S} \hat{R}^{-1} \hat{S}^{T} & =0 \\
D_{*}+\hat{R}^{-1}\left(\hat{S}^{T}+\hat{B}^{T} P\right) & =0
\end{aligned}
$$

where $\tilde{\hat{A}}=\hat{A}-\hat{B} \hat{R}^{-1} \hat{S}^{T}$. In Theorem 1 the conditions for existence of a unique stabilizing positive definite solution to the ARE were defined. First we have the assumption

$$
\left(\begin{array}{cc}
\hat{Q} & \hat{S} \\
\hat{S}^{T} & \hat{R}
\end{array}\right) \succ 0
$$

However, since the coordinate transformation matrix $\Pi$ has full column rank it follows that

$$
\left(\begin{array}{cc}
Q & S \\
S^{T} & R
\end{array}\right) \succ 0 \Rightarrow\left(\begin{array}{cc}
\hat{Q} & \hat{S} \\
\hat{S}^{T} & \hat{R}
\end{array}\right) \succ 0
$$

In some cases it is not desired to penalize $x_{2}$. In these cases the weight matrix is given by

$$
\left(\begin{array}{cc}
\hat{Q} & \hat{S} \\
\hat{S}^{T} & \hat{R}
\end{array}\right)=\Pi^{T}\left(\begin{array}{ccc}
Q_{11} & 0 & S_{1} \\
0 & 0 & 0 \\
S_{1}^{T} & 0 & R
\end{array}\right) \Pi=\left(\begin{array}{cc}
Q_{11} & S_{1} \\
S_{1}^{T} & R
\end{array}\right)
$$

which means that if the weight matrix for $x_{1}$ and $u$ is positive definite, the weight matrix for the composite system (25) (29) will also be so. Given the assumption above, we know from Theorem 1 that (29) has a unique positive definite stabilizing solution if and only if

$$
(\hat{A}, \hat{B})=\left(A_{11}-A_{12} A_{22}^{-1} A_{21}, B_{1}-A_{12} A_{22}^{-1} B_{2}\right)
$$

is stabilizable.

Remark 2: Stabilizability of (31) is equivalent to stabilizability in descriptor sense [14] of the linearization of the descriptor system (4) if the system is of index one, which is assumed in this paper. This can be shown by applying the stabilizability test given in [14]

$$
\operatorname{rank}\{(s E-A \quad B)\}=n, \quad \forall s \in \mathbb{C}^{+}, s \text { finite }
$$

where $\mathbb{C}^{+}$denotes the closed right half plane.

The higher order terms of $V\left(x_{1}\right)$ and $u_{*}\left(x_{1}\right)$ are obtained from (12). In (12) only the series expansion coefficients of the different functions are included and it is therefore possible to replace these functions with the series expansion coefficients of $\hat{f}_{1 h}\left(x_{1}, u\right)$ and $\hat{L}_{h}\left(x_{1}, u\right)$, i.e.,

$$
\begin{aligned}
& V_{x_{1}}^{(m)}\left(x_{1}\right) \hat{A}_{c} x_{1}=-\sum_{k=3}^{m-1} V_{x_{1}}^{(k)}\left(x_{1}\right) \hat{B} u_{*}^{(m-k+1)}\left(x_{1}\right) \\
& -\sum_{k=2}^{m-1} V_{x_{1}}^{(k)}\left(x_{1}\right) \hat{f}_{1 h}^{(m-k+1)}\left(x_{1}, u_{*}\right) \\
& -2 \sum_{k=2}^{\left\lfloor\frac{m-1}{2}\right\rfloor} u_{*}^{(k)}\left(x_{1}\right)^{T} \hat{R} u_{*}^{(m-k)}\left(x_{1}\right) \\
& -u_{*}^{(m / 2)}\left(x_{1}\right)^{T} \hat{R} u_{*}^{(m / 2)}\left(x_{1}\right)-\hat{L}_{h}^{(m)}\left(x_{1}, u_{*}\right)
\end{aligned}
$$

where $m=3,4, \ldots, \hat{A}_{c}=\hat{A}-\hat{B} D_{*}$, and the terms $u^{(m / 2)}$ are to be omitted if $m$ is odd. The corresponding expression for the series expansion of the feedback law is

$$
\begin{aligned}
& u_{*}^{(k)}\left(x_{1}\right)=-\frac{1}{2} \hat{R}^{-1}\left\{V_{x_{1}}^{(k+1)}\left(x_{1}\right) \hat{B}\right. \\
& \left.+\sum_{i=1}^{k-1} V_{x_{1}}^{(k-i+1)}\left(x_{1}\right) \hat{f}_{1 h, u}^{(i)}\left(x_{1}, u_{*}\right)+\hat{L}_{h, u}^{(k)}\left(x_{1}, u_{*}\right)\right\}
\end{aligned}
$$

where for $k=2,3, \ldots$ In (32a) the terms $\hat{f}_{1 h}^{(i)}\left(x_{1}, u_{*}\right)$ and $\hat{L}_{h}^{(i)}\left(x_{1}, u_{*}\right)$ are given by the corresponding terms in (26) and (28) and therefore can be expressed in terms of the series expansions of the original functions as

$$
\hat{f}_{1 h}^{(i)}\left(x_{1}, u_{*}\right)=f_{1 h}^{(i)}\left(x_{1}, \varphi_{*}, u_{*}\right)+A_{12} \varphi_{h, *}^{(i)}
$$

and

$$
\begin{aligned}
& \hat{L}_{h}^{(i)}\left(x_{1}, u_{*}\right)=L_{h}^{(i)}\left(x_{1}, \varphi_{*}, u_{*}\right)+2 x_{1}^{T} Q_{12} \varphi_{h, *}^{(i-1)} \\
& +2\left(\varphi_{*}^{(1)}\right)^{T} Q_{22} \varphi_{h, *}^{(i-1)}+2 \sum_{k=2}^{[(i-1) / 2]}\left(\varphi_{h, *}^{(k)}\right)^{T} Q_{22} \varphi_{h, *}^{(i-k)} \\
& +\left(\varphi_{h, *}^{(i / 2)}\right)^{T} Q_{22} \varphi_{h, *}^{(i / 2)}+2 \sum_{k=1}^{i-2}\left(u_{*}^{(k)}\right)^{T} S_{2} \varphi_{h, *}^{(i-k)}
\end{aligned}
$$

where $\varphi_{*}=\varphi\left(x_{1}, u_{*}\right)$ and $\varphi_{h, *}=\varphi_{h}\left(x_{1}, u_{*}\right)$. In (32), the series expansion coefficients of the functions $\hat{f}_{1 h}$ and $\hat{L}_{h}$ were needed. These were easily obtained as (33a) and (33b) respectively. However, in the expression for the optimal control signal (32b), the derivatives of $\hat{f}_{1}\left(x_{1}, u\right)$ and $\hat{L}\left(x_{1}, u\right)$ with respect to $u$ are needed. These expressions become

$$
\begin{aligned}
& \hat{f}_{1 h, u}^{(i)}\left(x_{1}, u_{*}\right)=f_{1 h, u}\left(x_{1}, \varphi_{*}, u_{*}\right)^{(i)} \\
& +\sum_{j=1}^{i} f_{1 h, x_{2}}^{(j)}\left(x_{1}, \varphi_{*}, u_{*}\right) \varphi_{u, *}^{(i-j)}+A_{12} \varphi_{h, u, *}^{(i)}
\end{aligned}
$$

where $\varphi_{h, u, *}^{(i)}=\varphi_{h, u}^{(i)}\left(x_{1}, u_{*}\right), \varphi_{u, *}^{(i)}=\varphi_{u}^{(i)}\left(x_{1}, u_{*}\right)$ and

$$
\begin{aligned}
& \hat{L}_{h, u}^{(k)}\left(x_{1}, u_{*}\right)=L_{h, u}^{(k)}\left(x_{1}, \varphi_{*}, u_{*}\right) \\
& +\sum_{j=2}^{k} L_{h, x_{2}}^{(j)}\left(x_{1}, \varphi_{*}, u_{*}\right) \varphi_{u, *}^{(k-j)}+2 x_{1}^{T} Q_{12} \varphi_{h, u, *}^{(k-1)} \\
& +2 \varphi_{*}^{(1)} Q_{22} \varphi_{h, u, *}^{(k-1)}-2 B_{2}^{T} A_{22}^{T} Q_{22} \varphi_{h, *}^{(k)} \\
& +\sum_{j=1}^{k-2}\left(\varphi_{h, u, *}^{(j)}\right)^{T} Q_{22} \varphi_{h, *}^{(k-j)}+\sum_{j=1}^{k-2}\left(\varphi_{h, *}^{(k-j)}\right)^{T} Q_{22} \varphi_{h, u, *}^{(j)} \\
& +2 S_{2} \varphi_{h, *}^{(k)}+2 \sum_{j=1}^{k-1} u_{*}^{(j)} S_{2} \varphi_{h, u, *}^{(k-j)}
\end{aligned}
$$

Since $f_{1 h}(x, u), \varphi_{h}\left(x_{1}, u\right)$ and $L_{h}(x, u)$ are power series of degree two, two and three respectively, and

$$
\varphi^{(m)}\left(x_{1}, u_{*}\right)=\varphi\left(x_{1}, u_{*}^{(1)}+u_{*}^{(2)}+\ldots+u_{*}^{(m)}\right)
$$

and as for the state space case, the right-hand side of (32a) only depends on the sequence (14) while the right-hand 
side of (32b) only depends on (15). So by consecutively calculating the terms of the series

$$
V^{(2)}\left(x_{1}\right), u_{*}^{(1)}\left(x_{1}\right), \varphi^{(1)}\left(x_{1}, u_{*}^{(1)}\right), \ldots
$$

it is possible to generate the power series for $V\left(x_{1}\right), u_{*}\left(x_{1}\right)$ and $\varphi\left(x_{1}, u_{*}\left(x_{1}\right)\right)$.

In the sequence above, it can be seen that it is unnecessary to calculate orders of $\varphi\left(x_{1}, u\right)$ higher than the desired order of the approximation of $u_{*}\left(x_{1}\right)$. However, as can be seen in (21) and (23), it is possible to compute arbitrarily high orders of $\varphi\left(x_{1}, u\right)$ in advance.

\section{EXAMPLE}

In order to illustrate the method we will study a small example. The system dynamics is given by the set of differential and algebraic equations

$$
\begin{aligned}
\dot{w}_{1} & =w_{2} \\
\dot{w}_{2} & =e^{w_{3}}-1+\frac{1}{2} u \\
0 & =w_{1}-\arcsin \left(1-e^{w_{3}}+\frac{1}{2} u\right)
\end{aligned}
$$

The physical interpretation of (35) is a Phase-Locked Loop circuit (PLL) which is used to control an oscillator so that it maintains a constant phase angle relative to a reference signal. The objective is to find the feedback law (7) which minimizes the cost function (2) with the cost function chosen as

$$
\begin{aligned}
& L\left(x_{1}, x_{2}, u\right) \\
& =\frac{1}{2} w_{1}^{2}+2 w_{1} w_{2}+w_{2}^{2}+w_{1}\left(-e^{w_{3}}+1+\frac{1}{2} u\right)+\frac{1}{2} u^{2}
\end{aligned}
$$

When the variables are grouped according to $x_{1}=$ $\left(w_{1}, w_{2}\right)^{T}$ and $x_{2}=w_{3}$, the system is on the form (4), with

$$
\begin{aligned}
& f_{1}\left(x_{1}, x_{2}, u\right)=\left(\begin{array}{c}
w_{2} \\
e^{w_{3}}-1+\frac{1}{2} u
\end{array}\right) \\
& f_{2}\left(x_{1}, x_{2}, u\right)=w_{1}-\arcsin \left(1-e^{w_{3}}+\frac{1}{2} u\right)
\end{aligned}
$$

and

$$
E=\left(\begin{array}{lll}
1 & 0 & 0 \\
0 & 1 & 0 \\
0 & 0 & 0
\end{array}\right)
$$

Expressing the descriptor system on the form (5a) by computing the power series expansion of $f_{1}\left(x_{1}, x_{2}, u\right)$ and $f_{2}\left(x_{1}, x_{2}, u\right)$ around the origin yields the first order terms

$$
A=\left(\begin{array}{lll}
0 & 1 & 0 \\
0 & 0 & 1 \\
1 & 0 & 1
\end{array}\right), \quad B=\left(\begin{array}{c}
0 \\
1 / 2 \\
-1 / 2
\end{array}\right)
$$

and the higher order terms of order up till three as

$$
\left(\begin{array}{c}
f_{1 h} \\
f_{2 h}
\end{array}\right)=\left(\begin{array}{c}
0 \\
1 / 2 w_{3}^{2}+1 / 6 w_{3}^{3} \\
1 / 2 w_{3}^{2}+1 / 3 w_{3}^{3}-1 / 4 u w_{3}^{2}+1 / 8 u^{2} w_{3}-1 / 48 u^{3}
\end{array}\right)
$$

As can be seen in (38) the matrix $A_{22}=1$, i.e., it is nonsingular. This guarantees that it is possible to compute the function $\varphi\left(x_{1}, u\right)$ using the implicit function theorem. From (21) the first order term of $\varphi\left(x_{1}, u\right)$ is obtained as

$$
\varphi^{(1)}\left(x_{1}, u\right)=-w_{1}+\frac{1}{2} u
$$

and the higher order terms of $\varphi_{h}\left(x_{1}, u\right)$ to order three are then recursively computed using (23) as

$$
\begin{aligned}
\varphi_{h}\left(x_{1}, u\right) & =-1 / 2 w_{1}^{2}+1 / 2 w_{1} u-1 / 8 u^{2} \\
& -1 / 6 w_{1}^{3}+1 / 2 u w_{1}^{2}-1 / 4 u^{2} w_{1}+1 / 24 u^{3}
\end{aligned}
$$

Equation (24) together with (40) will then define the local state variable change as

$$
\left(\begin{array}{l}
w_{1} \\
w_{2} \\
w_{3} \\
u
\end{array}\right)=\left(\begin{array}{ccc}
1 & 0 & 0 \\
0 & 1 & 0 \\
-1 & 0 & 1 / 2 \\
0 & 0 & 1
\end{array}\right)\left(\begin{array}{l}
w_{1} \\
w_{2} \\
u
\end{array}\right)
$$

The system matrices for the composite system (25), i.e., $\hat{A}$ and $\hat{B}$, can then be computed as

$$
\hat{A}=\left(\begin{array}{cc}
0 & 1 \\
-1 & 0
\end{array}\right), \quad \hat{B}=\left(\begin{array}{l}
0 \\
1
\end{array}\right)
$$

We also need the power series expansion of the cost function (36). Around the origin this can be computed to the fourth order as

$$
L=\left(\begin{array}{c}
w_{1} \\
w_{2} \\
w_{3} \\
u
\end{array}\right)^{T}\left(\begin{array}{ccc}
Q_{11} & Q_{12} & S_{1} \\
Q_{12}^{T} & Q_{22} & S_{2} \\
S_{1}^{T} & S_{2}^{T} & R
\end{array}\right)\left(\begin{array}{c}
w_{1} \\
w_{2} \\
w_{3} \\
u
\end{array}\right)-\frac{1}{2} w_{1} w_{3}^{2}-\frac{1}{6} w_{1} w_{3}^{3}
$$

where the cost matrix is

$$
\left(\begin{array}{ccc}
Q_{11} & Q_{12} & S_{1} \\
Q_{12}^{T} & Q_{22} & S_{2} \\
S_{1}^{T} & S_{2}^{T} & R
\end{array}\right)=\left(\begin{array}{cccc}
1 / 2 & 1 & -1 / 2 & 1 / 4 \\
1 & 1 & 0 & 0 \\
-1 / 2 & 0 & 0 & 0 \\
1 / 4 & 0 & 0 & 1 / 2
\end{array}\right)
$$

The matrix (45) is not positive definite but indefinite. However, if (42) is utilized on (45) as described in (27) the cost matrix for the composite system (17a) will become

$$
\left(\begin{array}{cc}
\hat{Q} & \hat{S} \\
\hat{S}^{T} & \hat{R}
\end{array}\right)=\left(\begin{array}{ccc}
3 / 2 & 1 & 0 \\
1 & 1 & 0 \\
0 & 0 & 1 / 2
\end{array}\right)
$$

which is positive definite.

From Section IV we know that the computations follow the order defined by the sequence

$$
V^{(2)}\left(x_{1}\right), u_{*}^{(1)}\left(x_{1}\right), \varphi_{*}^{(1)}\left(x_{1}\right), V^{(3)}\left(x_{1}\right), \ldots
$$

Since $(\hat{A}, \hat{B})$ is stabilizable and the cost matrix (46) is positive definite we know from Theorem 1 that the ARE (29a) has a unique stabilizing positive definite solution. The first terms in the approximation can then be computed as described in (13) whereby we obtain

$$
\begin{aligned}
V^{(2)}\left(x_{1}\right) & =\left(\begin{array}{l}
w_{1} \\
w_{2}
\end{array}\right)^{T}\left(\begin{array}{cc}
1 & 1 / 2 \\
1 / 2 & 1
\end{array}\right)\left(\begin{array}{l}
w_{1} \\
w_{2}
\end{array}\right) \\
u_{*}^{(1)}\left(x_{1}\right) & =\left(\begin{array}{ll}
-1 & -2
\end{array}\right)\left(\begin{array}{l}
w_{1} \\
w_{2}
\end{array}\right)
\end{aligned}
$$

The corresponding closed loop system matrix will then be

$$
\hat{A}_{c}=\left(\begin{array}{cc}
0 & 1 \\
-2 & -2
\end{array}\right)
$$

which has the eigenvalues $\lambda=-1 \pm 1 i$. Now it is possible to compute $\varphi_{*}^{(1)}\left(x_{1}\right)$ and $\varphi_{*, h}^{(2)}\left(x_{1}\right)$ as

$\varphi_{*}^{(1)}\left(x_{1}\right)=-\frac{3}{2} w_{1}-w_{2}, \quad \varphi_{h, *}^{(2)}\left(x_{1}\right)=-\frac{9}{8} w_{1}^{2}-\frac{3}{2} w_{1} w_{2}-\frac{1}{2} w_{2}^{2}$ 
In order to calculate the third order term in the approximation of $V\left(x_{1}\right),(32 \mathrm{a})$ is used. With $m=3$ the expression becomes

$$
\begin{aligned}
& V_{x_{1}}^{(3)}\left(x_{1}\right) \hat{A}_{c} x_{1}= \\
& \quad-V_{x_{1}}^{(2)}\left(x_{1}\right) \hat{f}_{1 h}^{(2)}\left(x_{1}, u_{*}\right)-\hat{L}_{h}^{(3)}\left(x_{1}, u_{*}\right)=0
\end{aligned}
$$

since by using (33) we obtain

$$
\hat{f}_{1 h}^{(2)}\left(x_{1}, u_{*}^{(1)}\right)=\left(\begin{array}{l}
0 \\
0
\end{array}\right), \quad \hat{L}_{h}^{(3)}\left(x_{1}, u_{*}^{(1)}\right)=0
$$

Solving (49) then results in

$$
V^{(3)}\left(x_{1}\right)=0
$$

Remark 3: The problem is symmetric in the sense that the same problem is obtained if $w$ is replaced by $-w$. Therefore, it is natural that $V^{(k)}\left(x_{1}\right)=0$ for odd $k$.

The next step is to solve for $u_{*}^{(2)}\left(x_{1}\right)$. This is done by letting $k=2$ in (32b) which gives

$$
\begin{aligned}
& u_{*}^{(2)}\left(x_{1}\right)=-\frac{1}{2} \hat{R}^{-1} \\
& \cdot\left\{V_{x_{1}}^{(3)}\left(x_{1}\right) \hat{B}+V_{x_{1}}^{(2)}\left(x_{1}\right) \hat{f}_{1 h, u}^{(1)}\left(x_{1}, u_{*}\right)+\hat{L}_{h, u}^{(2)}\left(x_{1}, u_{*}\right)\right\}
\end{aligned}
$$

The unknown terms in (52) are then computed by utilizing (34) and we obtain

$$
\hat{f}_{1 h, u}^{(1)}\left(x_{1}, u_{*}^{(1)}\right)=\left(\begin{array}{l}
0 \\
0
\end{array}\right), \quad \hat{L}_{h}^{(2)}\left(x_{1}, u_{*}^{(1)}\right)=0
$$

which together with the fact that $V^{(3)}\left(x_{1}\right)=0$ yields that also $u_{*}^{(2)}\left(x_{1}\right)=0$.

We will also calculate $V^{(4)}\left(x_{1}\right)$. Then $\varphi_{*}^{(2)}\left(x_{1}\right)$ and $\varphi_{h, *}^{(3)}\left(x_{1}\right)$ are needed. Since $u_{*}^{(2)}\left(x_{1}\right)=0$ we have that $\varphi_{*}^{(2)}\left(x_{1}\right)$ will equal $\varphi_{h, *}^{(2)}\left(x_{1}\right)$ while

$$
\varphi_{h, *}^{(3)}\left(x_{1}\right)=-\frac{23}{24} w_{1}^{3}-\frac{9}{4} w_{1}^{2} w_{2}-\frac{3}{2} w_{1} w_{2}^{2}-\frac{1}{3} w_{2}^{3}
$$

Again using (32a) leads to the expression

$$
\begin{array}{r}
V_{x_{1}}^{(4)}\left(x_{1}\right) \hat{A}_{c} x_{1}=-V_{x_{1}}^{(3)}\left(x_{1}\right) \hat{B} u_{*}^{(2)}-V_{x_{1}}^{(2)}\left(x_{1}\right) \hat{f}_{1 h}^{(3)}\left(x_{1}, u_{*}\right) \\
-V_{x_{1}}^{(3)}\left(x_{1}\right) \hat{f}_{1 h}^{(2)}\left(x_{1}, u_{*}\right)-\hat{L}_{h}^{(4)}\left(x_{1}, u_{*}\right)
\end{array}
$$

where

$$
\begin{aligned}
\hat{L}_{h}^{(4)}\left(x_{1}, u_{*}^{(1)}+u_{*}^{(2)}\right) & =-\frac{1}{6} w_{1}^{4} \\
V_{x_{1}}^{(2)}\left(x_{1}\right) \hat{f}_{1 h}^{(3)}\left(x_{1}, u_{*}^{(1)}+u_{*}^{(2)}\right) & =\frac{1}{6} w_{1}^{3}\left(w_{1}+2 w_{2}\right)^{2}
\end{aligned}
$$

and the other terms in (54) equal zero. The solution to (54) becomes

$$
V^{(4)}\left(x_{1}\right)=-\frac{1}{12} w_{1}^{4}
$$

The details of the calculation of $u_{*}^{(3)}\left(x_{1}\right)$ are omitted, but the result is $u_{*}^{(3)}\left(x_{1}\right)=0$.

In order to validate the solutions of the power series method we need the series expansions of the explicit solutions $u_{*}\left(x_{1}\right)$ and $V\left(x_{1}\right)$. The system (35) can be formulated on state space form as

$$
\begin{aligned}
& \dot{w}_{1}=w_{2} \\
& \dot{w}_{2}=-\sin \left(w_{1}\right)+u
\end{aligned}
$$

with the cost function (36) given as

$$
L\left(x_{1}, u\right)=\frac{1}{2} w_{1}^{2}+2 w_{1} w_{2}+w_{2}^{2}+w_{1} f\left(w_{1}\right)+\frac{1}{2} u^{2}
$$

By solving the HJB (8) the explicit expressions are given by

$$
\begin{aligned}
u_{*}\left(x_{1}\right) & =-w_{1}-2 w_{2} \\
V\left(x_{1}\right) & =2\left(1-\cos \left(w_{1}\right)\right)+w_{1} w_{2}+w_{2}^{2}
\end{aligned}
$$

For $u_{*}\left(x_{1}\right)$ we need not do any truncation since the exact solution is a polynomial in $x_{1}$ of order one. However, for $V\left(x_{1}\right)$ we compute the series expansions of (59) to the fourth order as

$$
V\left(x_{1}\right)=w_{1}^{2}+w_{1} w_{2}+w_{2}^{2}-\frac{1}{12} w_{1}^{4}
$$

A comparison between the power series expansions of the explicit solutions (58), (60) and the solutions to the power series method (47), (51), (56) shows that the same expressions are attained.

\section{CONCLUSIONS}

In this paper a method has been presented which makes it possible to calculate a power series solution to the HJB equation for descriptor systems. The solution is exact as long as no truncation of the power series is done. The first terms $u^{(1)}\left(x_{1}\right)$ and $V^{(2)}\left(x_{1}\right)$ are obtained by solving an algebraic Riccati equation. The higher order terms of $u_{*}\left(x_{1}\right)$ and $V\left(x_{1}\right)$ are then calculated recursively using systems of linear equations. The fundamental limits are that the linearization of the descriptor system around the origin must be stabilizable and have index one.

\section{REFERENCES}

[1] N. H. McClamroch, "Feedback stabilization of control systems described by a class of nonlinear differential-algebraic equations," Syst. Control Lett., vol. 15, no. 1, pp. 53-60, 1990.

[2] A. Kumar and P. Daoutidis, "Feedback regularization and control of nonlinear differential-algebraic-equation systems," AIChE Journal, vol. 42, no. 8, pp. 2175-2198, 1996.

[3] H. S. Wang, C. F. Yung, and F.-R. Chang, " $H_{\infty}$ control for nonlinear descriptor systems," IEEE Trans. Automat. Contr., vol. AC-47, no. 11, pp. 1919-1925, 2002.

[4] E. G. Al'brekht, "On the optimal stabilization of nonlinear systems," J. Appl. Math. Mech., vol. 25, no. 5, pp. 1254-1266, 1961.

[5] E. B. Lee and L. Markus, Foundations of Optimal Control Theory. New York: Wiley, 1967.

[6] D. L. Lukes, "Optimal regulation of nonlinear dynamical systems," SIAM J. Contr., vol. 7, no. 1, pp. 75-100, Feb. 1969.

[7] R. W. Beard, G. N. Saridis, and J. T. Wen, "Approximative solutions to the time-invariant Hamilton-Jacobi-Bellman equation," J. Optimiz. Theory App., vol. 96, no. 3, pp. 589-626, mar 1998.

[8] K. E. Brenan, S. Campbell, and L. R. Petzold, Numerical Solution of Initial-Value Problems in Differential-Algebraic Equations. SIAM, 1996.

[9] P. Kunkel and V. Mehrmann, "Analysis of over- and underdetermined nonlinear differential-algebraic systems with application to nonlinear control problems," Math. Control Signal, vol. 14, pp. 233-256, 2001

[10] G. Leitmann, The Calculus of Variations and Optimal Control. New York: Plenum Press, 1981.

[11] D. L. Lukes, "Stabilizability and optimal control," Funkcialaj Ekvacioj, vol. 11, no. 1, pp. 39-50, Sept. 1968.

[12] A. M. Lyapunov, The General Problem of the Stability of Motion. London: Taylor and Francis, 1992.

[13] L. Hörmander, An Introduction to Complex Analysis in Several Variables, ser. The University Series in Higher Mathematics. Princeton, NJ: D. Van Nostrand, 1966.

[14] L. Dai, Singular Control Systems, ser. Lecture Notes in Control and Information Sciences. Berlin, New York: Springer-Verlag, 1989. 